Acta Phys. Hung. A 19/1 (2004) 000-000

HEAVY ION

PHYSICS

\title{
Hadron attenuation at HERMES and Jefferson Lab
}

\author{
T. Falter, W. Cassing, K. Gallmeister and U. Mosel ${ }^{1}$ \\ ${ }^{1}$ Institut für Theoretische Physik, \\ Universität Giessen, Germany
}

Received 7 February 2005

\begin{abstract}
We investigate the attenuation of hadrons in deep inelastic leptonnucleus scattering in the kinematical regime of the HERMES and Jefferson Lab experiments. The calculation is carried out in the framework of a BUU transport model. Our results indicate a strong influence of (pre)hadronic final state interactions on the observed multiplicity ratios.
\end{abstract}

Keywords: deep inelastic lepton-nucleus scattering, meson production, hadron formation

PACS: 24.10.-i,25.30.-c,13.60.Le

\section{Introduction}

In deep inelastic scattering experiments the reaction products hadronize long before they reach the detector. Thus by using elementary nucleon targets one cannot obtain information on the space-time picture of hadronization. A simple estimate of the hadron formation proper time via the hadronic radius $r_{h}$ yields hadron formation lengths of the order $\gamma \cdot r_{h}$ in the laboratory frame. At high energies the Lorentz factor $\gamma$ leads to formation lengths that may easily exceed typical nuclear dimensions. By using nuclear targets one, therefore, has the unique possibility to investigate the final-state interactions (FSI) of the prehadronic system and to study the dynamics of the hadronization process.

In the recent past the HERMES collaboration has carried out an extensive study of hadron production in deep inelastic lepton-nucleus scattering using 27.6 $\mathrm{GeV}$ and $12 \mathrm{GeV}$ positron beams [1, 2. The observed attenuation of hadrons -compared to a deuterium target- has basically led to two different interpretations: The authors of Refs. [3] assume that hadronization occurs far outside the nucleus and that the attenuation is caused by a partonic energy loss prior to hadronization. On the other hand color neutral prehadrons might form rather early after the initial deep inelastic scattering event and undergo FSI on their way out of the nucleus [ 
[4. 5] 6. 7. The latter effect should be even more pronounced in the kinematical regime of the ongoing Jefferson Lab experiment which uses a lower energy $(5.4 \mathrm{GeV})$ electron beam [8].

In Ref. [5] we have given a thorough theoretical investigation of hadron attenuation in lepton-nucleus scattering at HERMES and EMC energies in the framework of a semi-classical Boltzmann-Uehling-Uhlenbeck (BUU) transport model. In this work we apply our model (with the same parameter set) to lower energies, i.e. HERMES at $12 \mathrm{GeV}$ beam energy and the kinematical regime of the Jefferson Lab experiment.

\section{Model}

In Refs. [4, 5, 9] we have developed a method to combine a quantum mechanical coherent treatment of nuclear shadowing -as observed in high energy photonuclear reactions- with an incoherent coupled-channel description of the (pre)hadronic FSI using a BUU transport model. This is achieved by splitting the electron-nucleus reaction into two parts. In the first step the high-energy virtual photon interacts with a bound nucleon inside the nucleus and produces a final state which is determined by using the Lund Monte Carlo generators PythiA and Fritiof. In addition we account for nuclear effects such as binding energies, Fermi motion, Pauli-blocking of final state nucleons and nuclear shadowing. In the second step the final state is propagated through the nucleus within the coupled-channel transport model.

The strength of the shadowing effect strongly depends on the coherence lengths of the photon's hadronic fluctuations. The coherence length can be understood as the distance that the virtual photon travels as a hadronic fluctuation which has the quantum numbers of the photon, e.g. a vector meson. If the coherence length exceeds the mean-free path of the hadronic fluctuation inside the nucleus, the photonnucleus interaction will get shadowed just like an ordinary hadron-induced nuclear reaction. In Fig. [1 we show the probability distribution for nucleons inside a ${ }^{84} \mathrm{Kr}$ nucleus to participate in the primary photon-nucleon interaction. The kinematics of the virtual photon with momentum along the $z$-axis corresponds to that of a typical HERMES event. For such a photon the coherence length of the $\rho^{0}$-meson fluctuation is of the order of the nuclear radius and the nucleons on the front side of the ${ }^{84} \mathrm{Kr}$ nucleus shadow the downstream nucleons.

The photon-nucleon interaction leads to the excitation of one or more strings which fragment into color-neutral prehadrons due to the creation of quark-antiquark pairs from the vacuum. As discussed in Ref. [ [6] the production time of these prehadrons is very short. For simplicity we set the production time to zero in our numerical realization. These prehadrons are then propagated using our coupledchannel transport theory.

After a formation time, which we assume to be a constant $\tau_{f}$ in the restframe of the hadron, the hadronic wave function has built up and the reaction products propagate and interact like usual hadrons. The prehadronic cross sections $\sigma^{*}$ during 


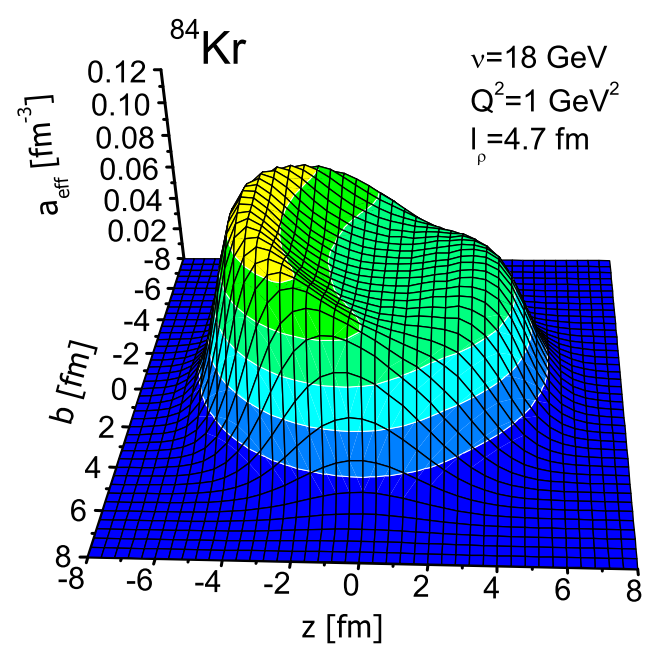

Fig. 1. Profile function for shadowing. Shown is the probability distribution for the interaction of an incoming photon (from left) with given virtuality $Q^{2}$ and energy $\nu$ with nucleons in a ${ }^{84} \mathrm{Kr}$ nucleus.

the formation time are determined by a simple constituent quark model

$$
\begin{aligned}
\sigma_{\text {prebaryon }}^{*} & =\frac{n_{\text {org }}}{3} \sigma_{\text {baryon }}, \\
\sigma_{\text {premeson }}^{*} & =\frac{n_{\text {org }}}{2} \sigma_{\text {meson }},
\end{aligned}
$$

where $n_{\text {org }}$ denotes the number of (anti-)quarks in the prehadron stemming from the beam or target. As a consequence the prehadrons that solely contain (anti-)quarks produced from the vacuum in the string fragmentation do not interact during $\tau_{f}$. Using this recipe the total effective cross section of the final state rises like in the approach of Ref. [10] each time when a new hadron has formed.

The FSI of the reaction products are described within our BUU transport model. The latter is based on a set of generalized transport equations for each particle species $i$,

$$
\left(\frac{\partial}{\partial t}+\vec{\nabla}_{\vec{p}} H \vec{\nabla}_{\vec{r}}-\vec{\nabla}_{\vec{r}} H \vec{\nabla}_{\vec{p}}\right) F_{i}(\vec{r}, \vec{p}, \mu ; t)=I_{\text {coll }}\left(\left\{F_{j}\right\}\right)
$$

where $H$ is a relativistic Hamilton function which contains a mean-field potential in case of baryons. The transport equations (2) describe the time-evolution of the spectral phase-space densities $F_{i}$ that are coupled via the collision integral $I_{\text {coll }}$. The latter accounts for changes in the spectral phase-space density due to particle creation and annihilation in binary collisions. This means that the final hadron in 
an electron-nucleus reaction does not necessarily need to be produced in the primary virtual photon-nucleon interaction but can be created later on via side-feeding in the FSI. We note that this (probabilistic) coupled-channel treatment of the FSI goes far beyond the usual single-channel Glauber approach.

\section{Results}

Due to our coupled channel-treatment of the FSI the (pre)hadrons might not only be absorbed in the nuclear medium but can produce new particles in an inelastic interaction, thereby shifting strength from the high to the low energy part of the hadron spectrum. In addition our event-by-event simulation allows us to account for all kinematic cuts and the acceptance of the detector. In Ref. [ 5] we have demonstrated that our calculations are in excellent agreement with the experimental HERMES data [2] taken on various nuclear targets at a beam energy $E_{\text {beam }}=27.6$ $\mathrm{GeV}$ if one assumes a formation time $\tau_{f}=0.5 \mathrm{fm} / \mathrm{c}$ for all hadron species. As an example we show in Fig. 2 the multiplicity ratios

$$
R_{M}^{h}\left(z_{h}, \nu\right)=\frac{\left.\frac{N_{h}\left(z_{h}, \nu\right)}{N_{e}(\nu)}\right|_{A}}{\left.\frac{N_{h}\left(z_{h}, \nu\right)}{N_{e}(\nu)}\right|_{D}}
$$

for identified hadrons on a ${ }^{84} \mathrm{Kr}$ target. Here $N_{h}$ is the yield of semi-inclusive hadrons in a given $\left(z_{h}, \nu\right)$-bin and $N_{e}$ the yield of inclusive deep inelastic scattering leptons in the same $\nu$-bin. For the deuterium target, i.e. the nominator of Eq. (3), we simply use the isospin averaged results of a proton and a neutron target. Thus in the case of deuterium we neglect the FSI of the produced hadrons and also the effect of shadowing and Fermi motion.

In Fig. 3] we show that our approach is also capable to describe the observed multiplicities of charged hadrons in the HERMES experiment at $E_{\text {beam }}=12 \mathrm{GeV}$. This success suggests that our model can also be applied for the electron beam energies that will be used at Jefferson Lab.

In Fig. 4 we present our predictions for the multiplicity ratios of identified hadrons at $5 \mathrm{GeV}$ electron beam energy. Besides the considerably lower beam energy used at Jefferson Lab the major difference to the HERMES experiment is the much larger geometrical acceptance of the CLAS detector. The latter leads to an increased detection of low energy secondary particles that are produced in the FSI and that lead to a strong increase of the multiplicity ratio at low fractional hadron energies $z_{h}=E_{h} / \nu$. In addition, the relatively small average photon energy leads to a visible effect of Fermi motion on the multiplicity ratio of more massive particles. Since the virtual photon cannot produce antikaons without an additional strange meson, e.g. $\gamma^{*} N \rightarrow K \bar{K} N$, the maximum fractional energy $z_{h}$ is limited to 0.9 for antikaons. Because of the energy distribution in the three body final state and the finite virtuality of the photon - set by the kinematic cut $Q^{2}>1 \mathrm{GeV}^{2}-$ the maximum fractional energy of antikaons is further reduced. As a result, the $z_{h}$ 


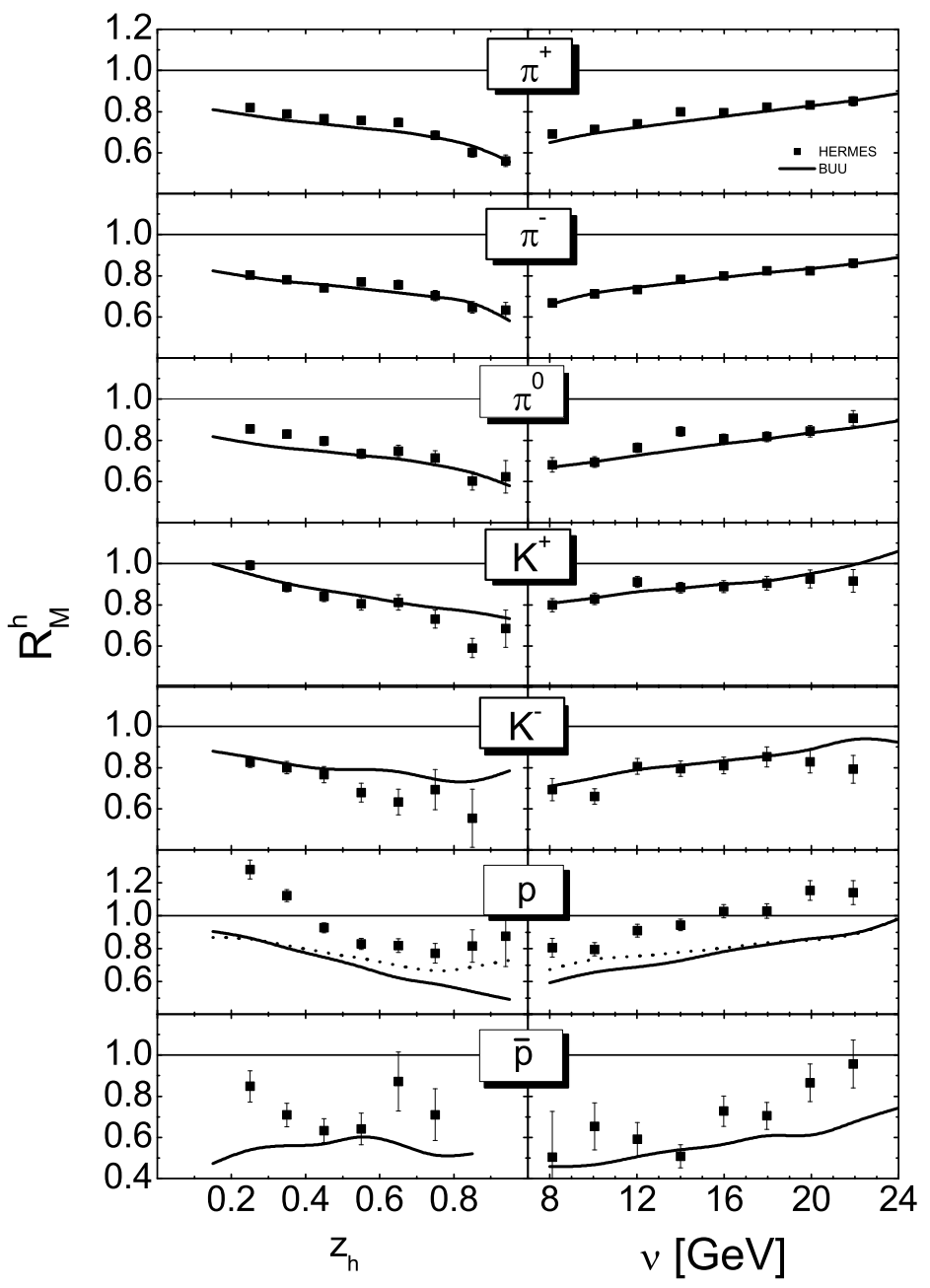

Fig. 2. Multiplicity ratios of $\pi^{ \pm, 0}, K^{ \pm}, p$ and $\bar{p}$ for a ${ }^{84} \mathrm{Kr}$ nucleus (when using a $27.6 \mathrm{GeV}$ positron beam at HERMES) as a function of the hadron energy fraction $z_{h}=E_{h} / \nu$ and the photon energy $\nu$. The solid line represents the result of a simulation, where we use the constituent quark concept (1) for the prehadronic cross sections and a formation time $\tau_{f}=0.5 \mathrm{fm} / c$. The dotted line in the proton spectrum indicates the result of a simulation where all $\gamma^{*} N$ events are created by PYTHIA. The data are taken from Ref. [2].

spectra for $K^{-}$and $\bar{K}^{0}$ in Fig. 4 do not exceed $z_{h} \approx 0.8$. For the same reason the production of antikaons with $z_{h}>0.2$ is reduced at the lower end of the photon spectrum. The Fermi motion in the nucleus enhances the yield of antikaons in these 


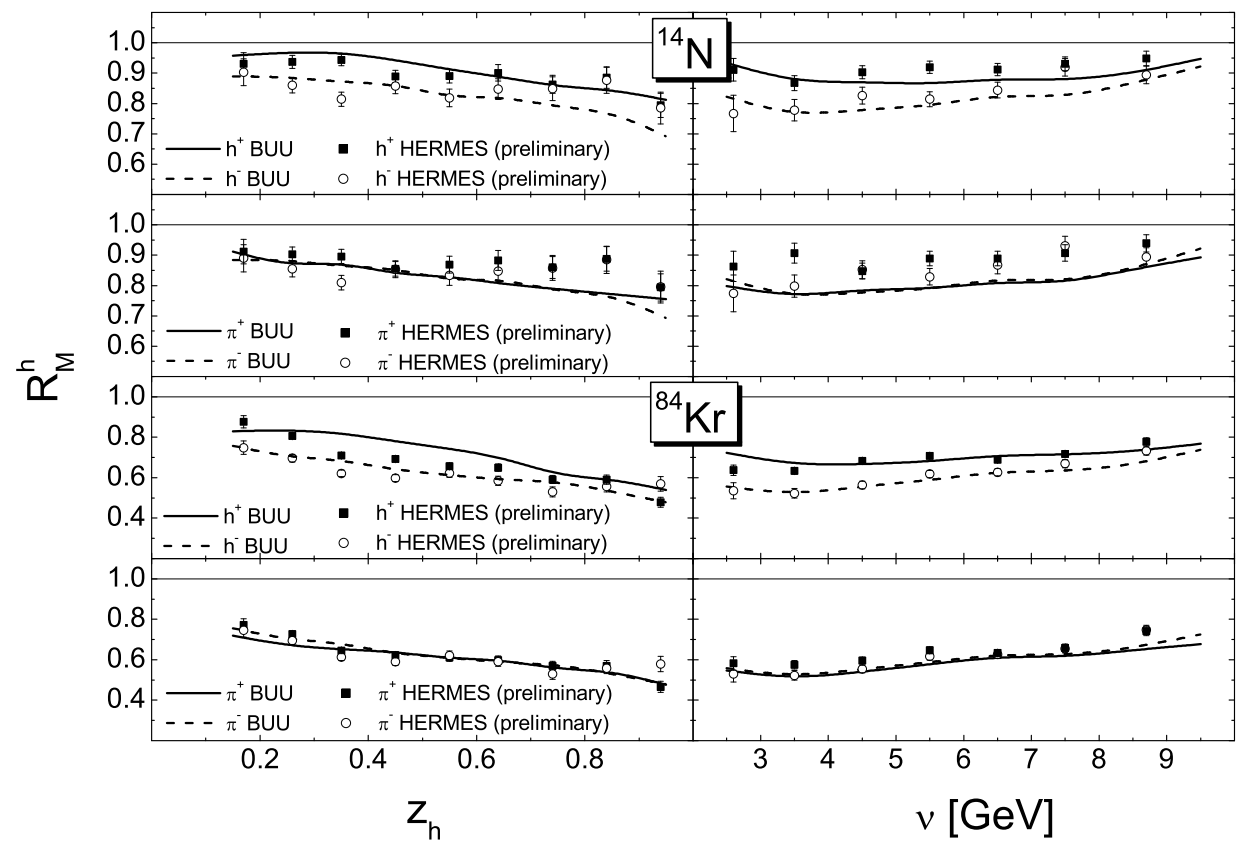

Fig. 3. Calculated multiplicity ratio of positively and negatively charged hadrons and pions for a ${ }^{14} \mathrm{~N}$ and ${ }^{84} \mathrm{Kr}$ target when using a $12 \mathrm{GeV}$ positron beam at HERMES. For the calculation we use the formation time $\tau_{f}=0.5 \mathrm{fm} / c$ and the constituent-quark concept (11) for the prehadronic cross sections. The data are taken from Ref. [11].

two extreme kinematic regions as can be seen by comparison with the dash-dotted line in Fig. 4 which represents the result of a calculation for ${ }^{54} \mathrm{Fe}$ where Fermi motion has been neglected. Certainly, the kaons can be produced in a two-body final state (e.g. $\gamma^{*} N \rightarrow K \Lambda$ ), however, the accompanying hyperon has a relatively large mass. Therefore, similar effects, although less pronounced, show also up for the kaons. Beside the effects of Fermi motion the multiplicity ratios of kaons and antikaons show the same features as for higher energies.

\section{Conclusions}

In this work we have presented a model that allows for a clean-cut separation of the initial state interactions of a high energy (virtual) photon -giving rise to nuclear shadowing- and the nuclear FSI of the reaction products. This allows to apply a coupled-channel transport code to perform realistic event-by-event simulations of high energy lepton-nucleus scattering and to account for experimental cuts and detector efficiencies. 


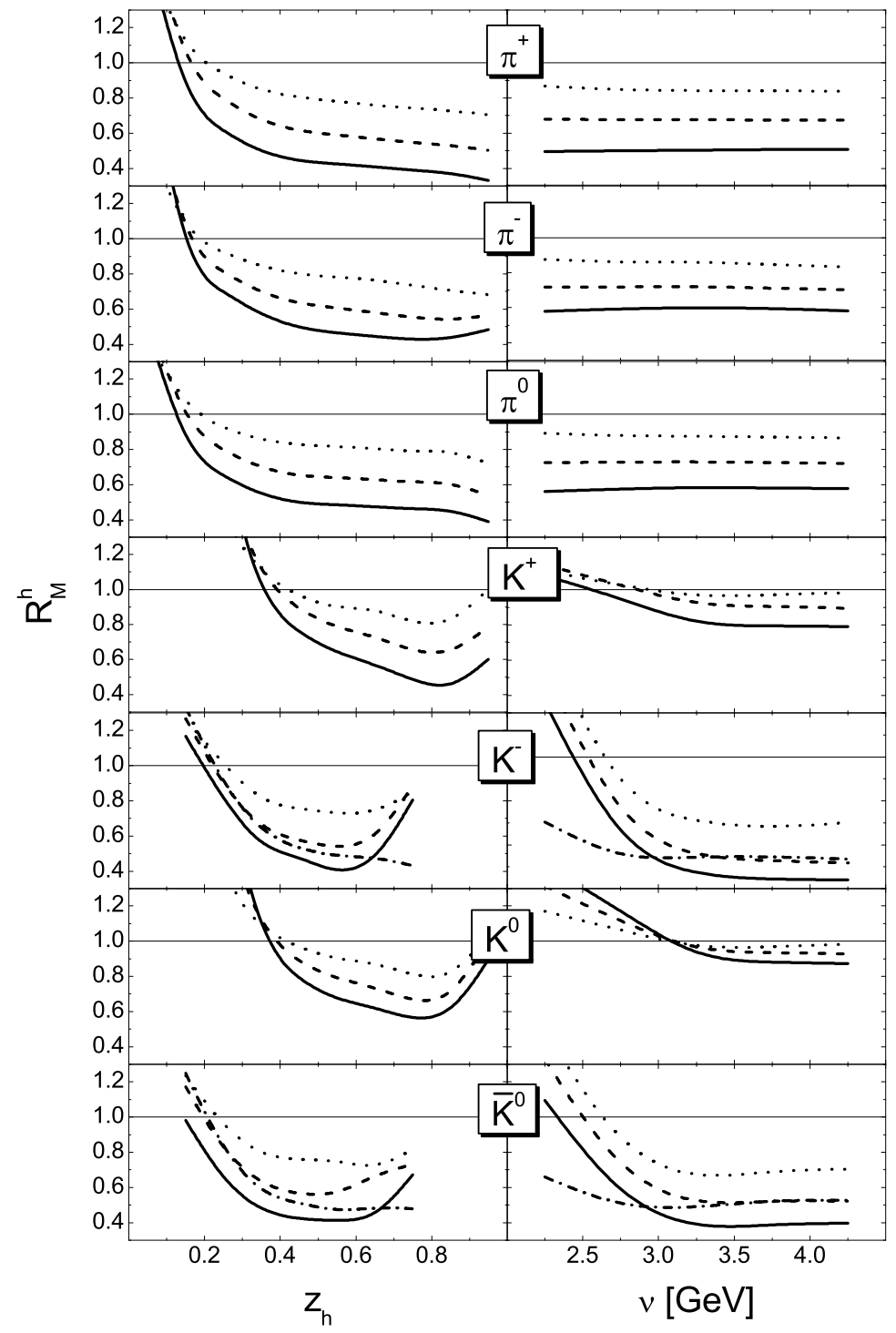

Fig. 4. Calculated multiplicity ratio of identified $\pi^{ \pm}, \pi^{0}, K^{ \pm}, K^{0}$ and $\bar{K}^{0}$ for ${ }^{12} \mathrm{C}$ (dotted lines), ${ }^{56} \mathrm{Fe}$ (dashed lines) and ${ }^{208} \mathrm{~Pb}$ nuclei (solid lines). The simulation has been done for a $5 \mathrm{GeV}$ electron beam and the CLAS detector. The dash-dotted line represents a calculation for ${ }^{56} \mathrm{Fe}$ without Fermi motion. In all calculations we use the formation time $\tau_{f}=0.5 \mathrm{fm} / c$ and the constituent-quark concept (11) for the prehadronic cross sections. 
From our comparison with the experimental data we conclude that a large part of the observed hadron attenuation at HERMES may be attributed to prehadronic FSI of the reaction products in the nuclear environment. Furthermore, we also expect a strong effect of these prehadronic FSI at the considerably lower Jefferson Lab energies.

Our implemented space-time picture of hadronization is still simplistic and needs improvements. In Ref. [12] we have therefore started to extract the fourdimensional prehadron production points from the Lund fragmentation routine JETSET in Pythia. This information will be used in future transport simulations of deep inelastic lepton-nucleus scattering.

\section{Acknowledgment}

This work has been supported by BMBF.

\section{References}

1. A. Airapetian et al. [HERMES Collaboration], Eur. Phys. J. C 20 (2001) 479.

2. A. Airapetian et al. [HERMES Collaboration], Phys. Lett. B 577 (2003) 37.

3. E. Wang and X.-N. Wang, Phys. Rev. Lett. 89 (2002) 162301; F. Arleo, Eur. Phys. J. C30 (2003) 213.

4. T. Falter, W. Cassing, K. Gallmeister and U. Mosel, Phys. Lett. B594 (2004) 61.

5. T. Falter, W. Cassing, K. Gallmeister and U. Mosel, Phys. Rev. C70 (2004) 054609 .

6. B. Z. Kopeliovich, J. Nemchik, E. Predazzi and A. Hayashigaki, Nucl. Phys. A740, 211 (2004).

7. A. Accardi, V. Muccifora and H.-J. Pirner, Nucl. Phys. A720 (2003) 131.

8. W. K. Brooks, Fizika B13 (2004) 321.

9. M. Effenberger and U. Mosel, Phys. Rev. C62 (2000) 014605; T. Falter and U. Mosel, Phys. Rev. C66 (2002) 024608; T. Falter, K. Gallmeister and U. Mosel, Phys. Rev. C67 (2003) 054606, Erratum-ibid. C68 (2003) 019903.

10. C. Ciofi degli Atti, B. Z. Kopeliovich, Eur. Phys. J. A17, 133 (2003).

11. P. Di Nezza [HERMES Collaboration], J. Phys. G. 30, S783 (2004).

12. K. Gallmeister and T. Falter, nucl-th/0502015 